\title{
Technique de la construction des Barrages en pierres lancées dans l'eau courante
}

\author{
CH. BLANCHET, E.I.H. \\ Ingénieur aux Ateliers Neyret-Beylier et Piccard-Pictet
}

(Suite)

\section{CHAPITRE III}

\section{TECHNIQUE PARTICULIERE DE CONSTRUCTION AVEC DES MATERIAUX TOUT-VENANT COUPURE DU RHONE A GENISSIAT \\ AVANT-PROPOS}

Nous avons exposé, précédemment, les laits essentiels relatifs à la constraction de massifs en pierres triées (pierres homogènes en grosseur)

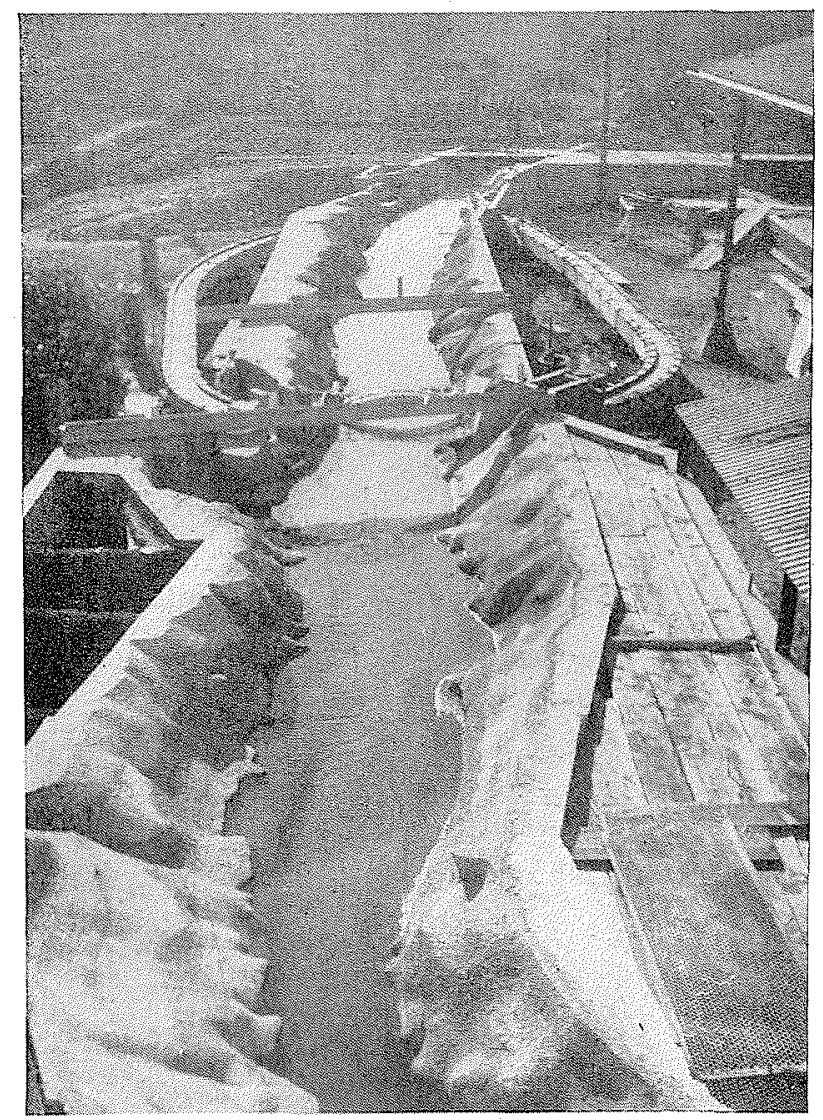

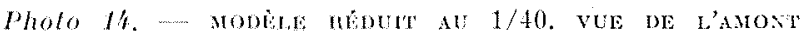

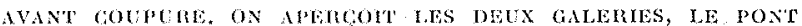
AMonf, chof Daval mavoue. que l'on jette dans le courant d'eau à barrer, de manière à élever progressivement la crête du massif uniformément sur toute la largeur du courant, suivant la méthode préconisée par M. Isbach, Directeur du Laboratoire Hydraulique de Moscou ( $)$.

Nous avons examiné ensuite les conséquences facheuses, pour l'élévation du massif, de l'emploi des matériaux tout-venant (mélange de pierres de grosseurs hétérogènes). Nous décrivons main.tenant ume technique spéciale de construction qui corrige et rend efficace le triage des matériaux tout-venant, qui deviennent alors en pratique, beaucoup plus intéressants que les matériaux triés.

Cette technique a été ẻlaborée sur les directives de la Cie Nationale du Rhòne, au cours des études sur modèle réduit de la coupure et dérivation du Rhòne à Génissiat qui avaient été confiées au Laboratoire Dauphinois d'Hydraulique « Neyrpic».

(1) Voir la Houlle Blanche $\mathrm{n}^{\circ}$ 6. 1946 .

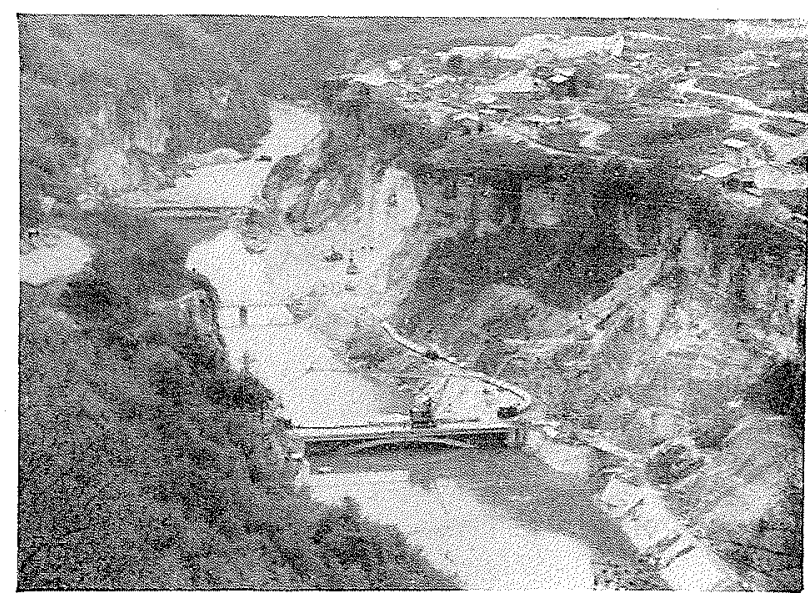

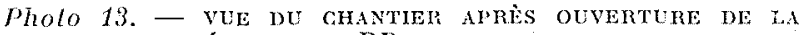
Galerie DE DÉRIVATION RD. LES Massifs DE COUPURE DERRIÈRE LES PONTS SONT TRİS PEU VISHBLES. 

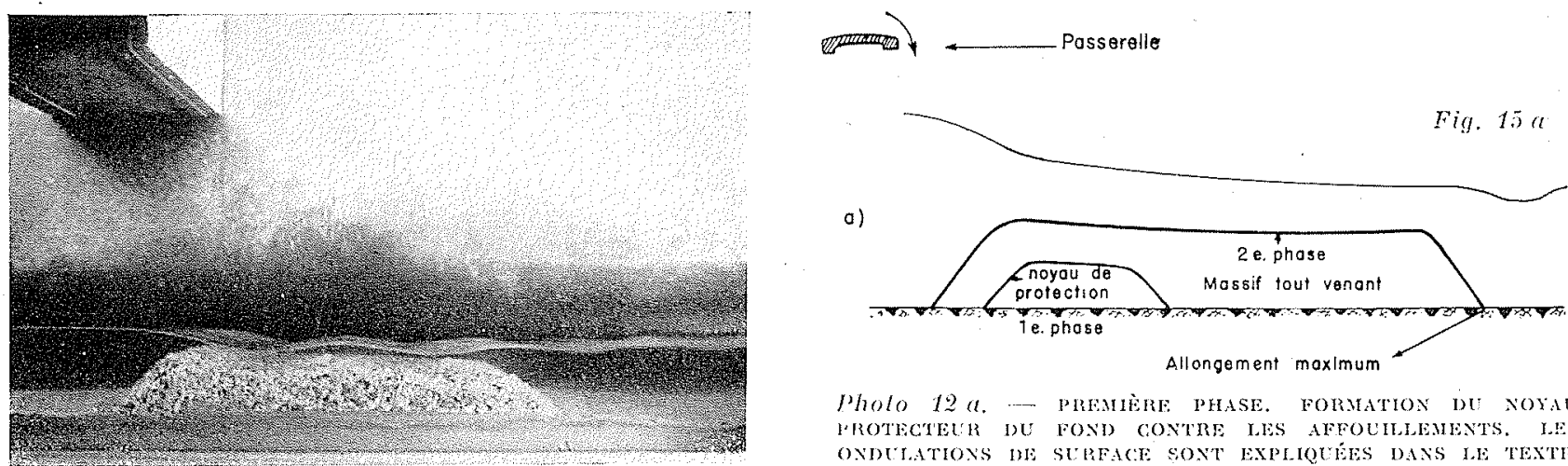

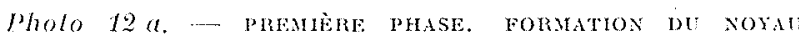
WROTECTELR DU FOND CONTRE LES AFFOULLEMEXTS MES ONDCHATONS DE SLPEACT SONT EXPLIOUGS DANS RE TEXTE,
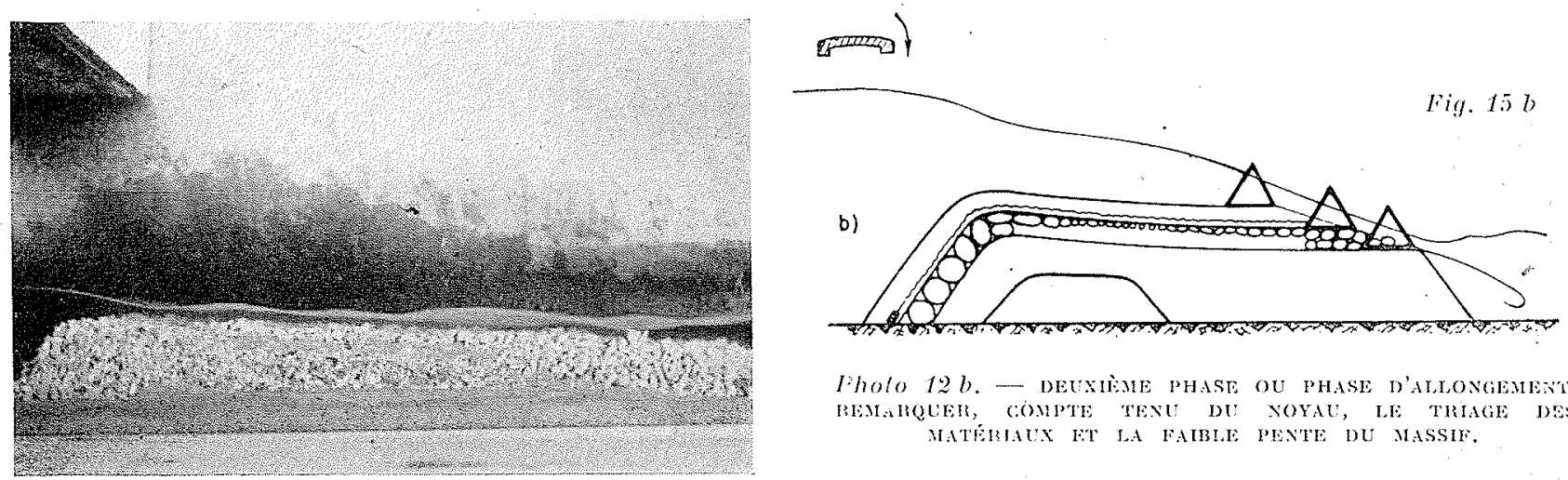

lholo $12 b$. - DEUKIVME PHASE OU PHASE D'ALHONGLMLA

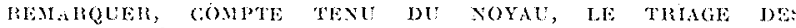
MATERIAUX FT LA FABLE PEXTE DU MASSIF,

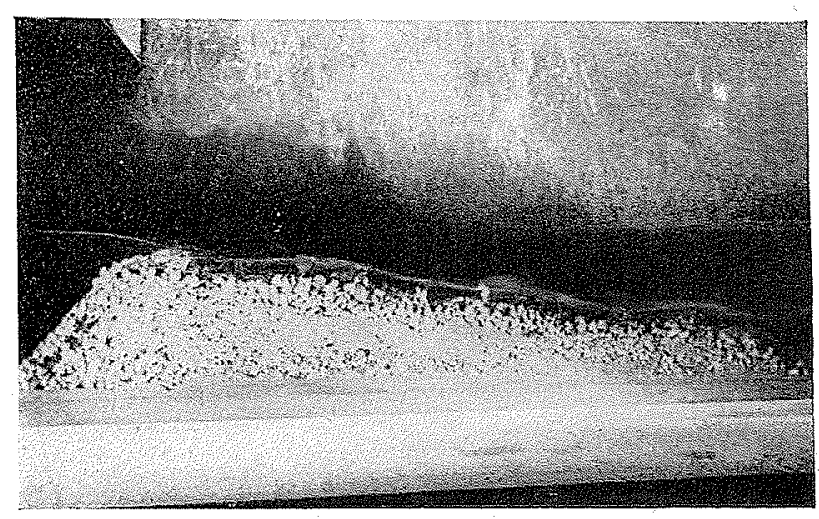

\section{METHODE DE CONSTRUCTION DU MASSIF}

Nous rappelons, tout d'abord, par la fig. 14(2), le triage el la répartition des matériaux toutvenant dans le massif construit normalemeni. puis les conséquences de ce triage à savoir que les gros éléments du tout-venant se soustraient à l'écoulement et ne joitent qu'un role de remplissage, tandis que les éléments fins se placeni sur la zone active du massif, qui, de ce fait, s'allonge beaucoup et s'éleve peu.

La technique que nous allons exposer permet d'inverser ces rôles, c'est-à-dire de stabiliser les

(2) Voir la Houlle Blunche n" (i. 1946

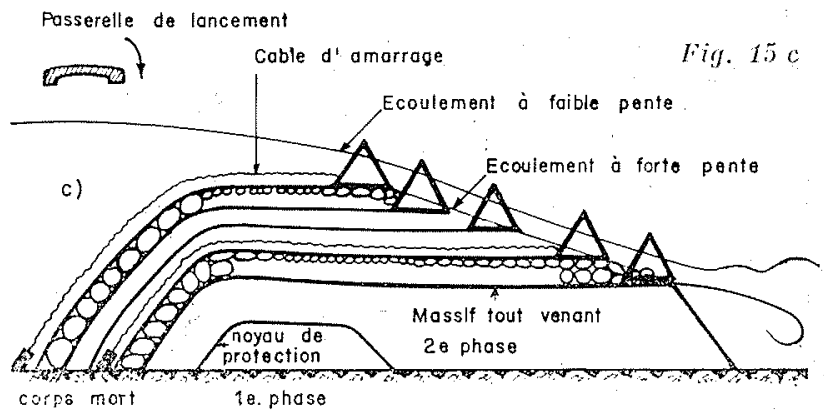

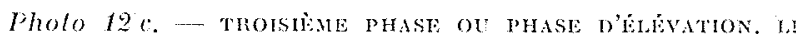

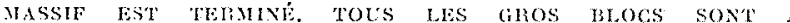

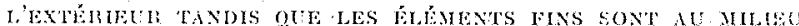
D) (O MASSIF.

gros éléments dans la zone d'écoulement et les bléments fins dans des zones oil ils ne serveni qu'au remplissage et à l'étanchéité.

Pour simplifier, nous exposerons directemeni le mode opératoire en ne le justifiant qu'au fur: et à mesure de l'exposé.

La construction comprend trois phases, dont la derniere est la principale.

Première phase ou noyau de protection (fig. 15 $a$, photo 12 a)....... Comme dans les mélhodes précédentes, on constitue d'abord le noyau de protection du lit de la rivière contre les alfoullements en jetant des matériaux de 


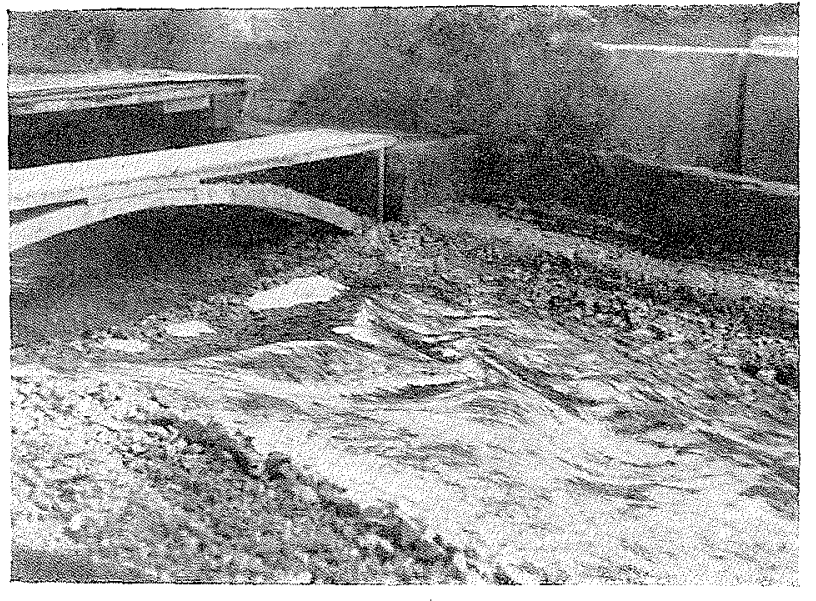

$15 a$

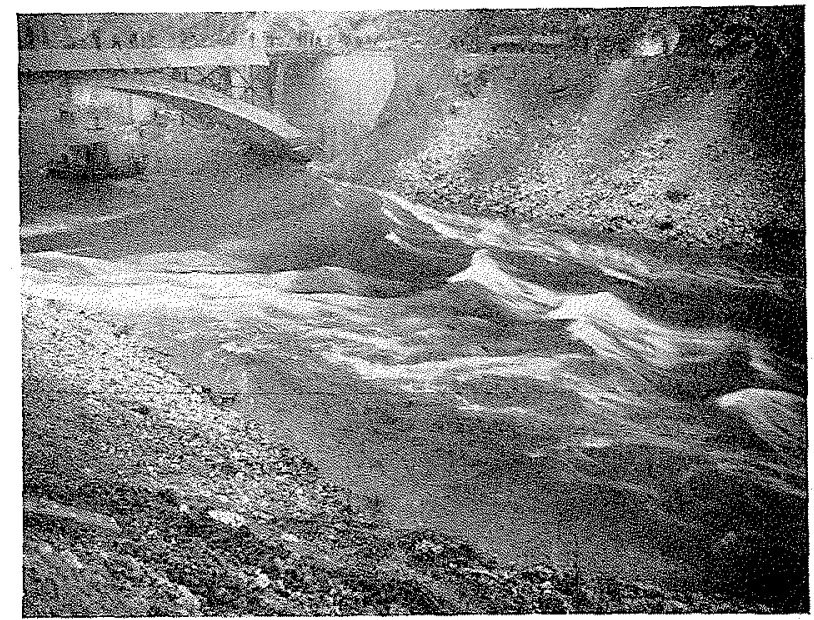

$15 \mathrm{~b}$

MASSH IYAL PENINAT SA PHASE D'ALLONGEMEYT

sur le modele redult a $1 / 40$ ( 15 a) et dans la réalite (1) $b$ ). Remarquer l'effet des berges rugueuses sur les ondulations de surface.

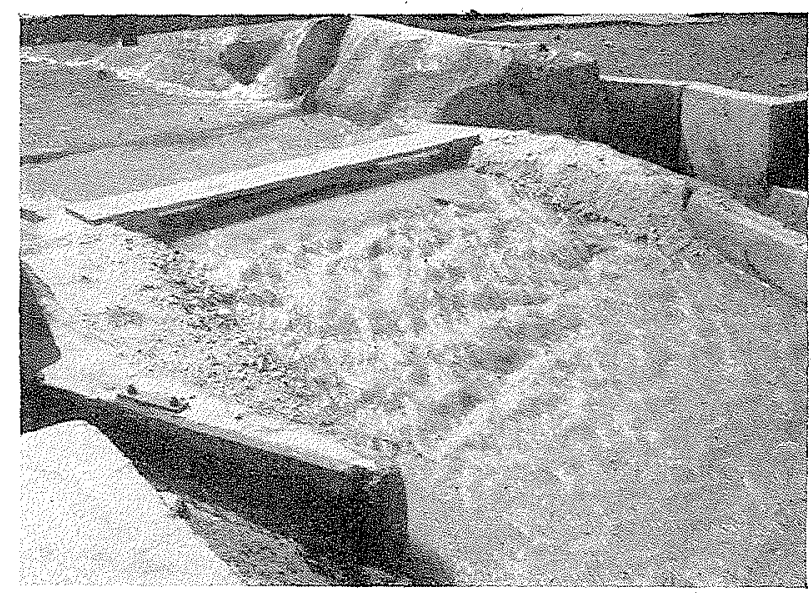

$16 a$

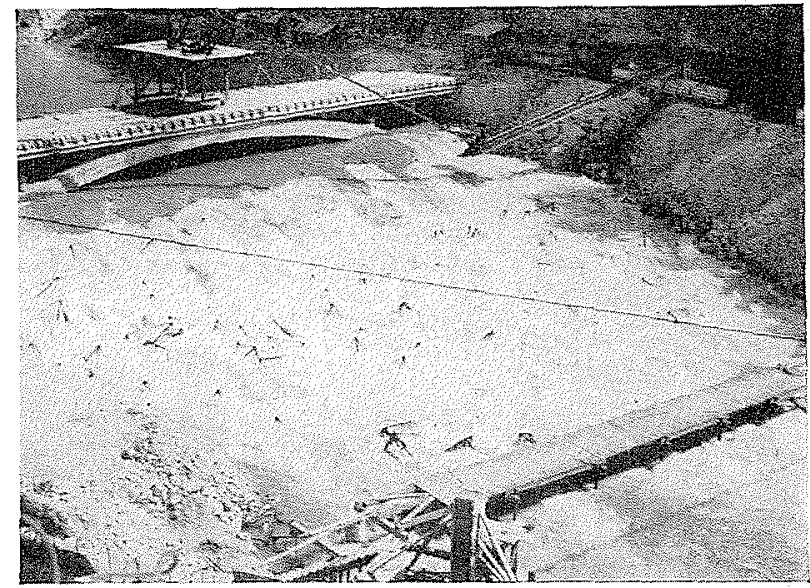

MASSIF AYAL TERMINÉ 16 a) modèlo

16 b) réalité

$16 \mathrm{~b}$

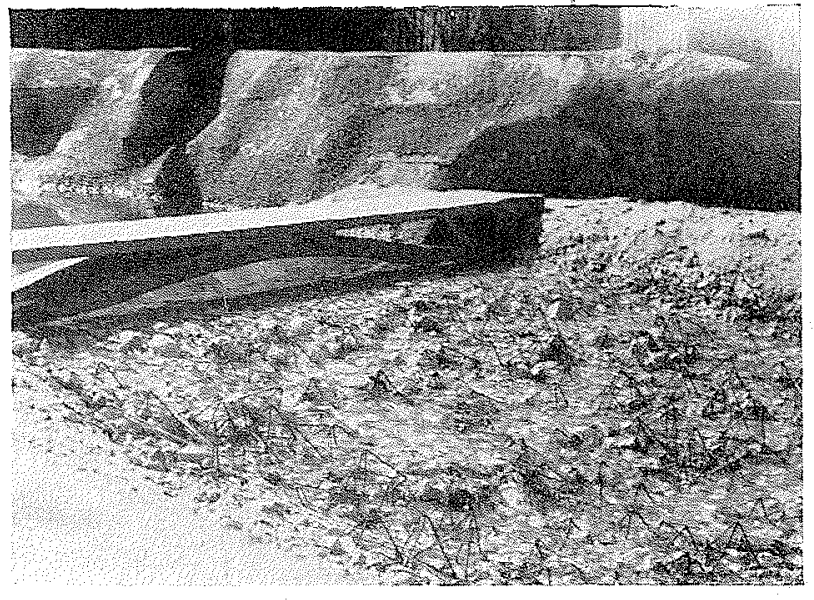

$17 a$

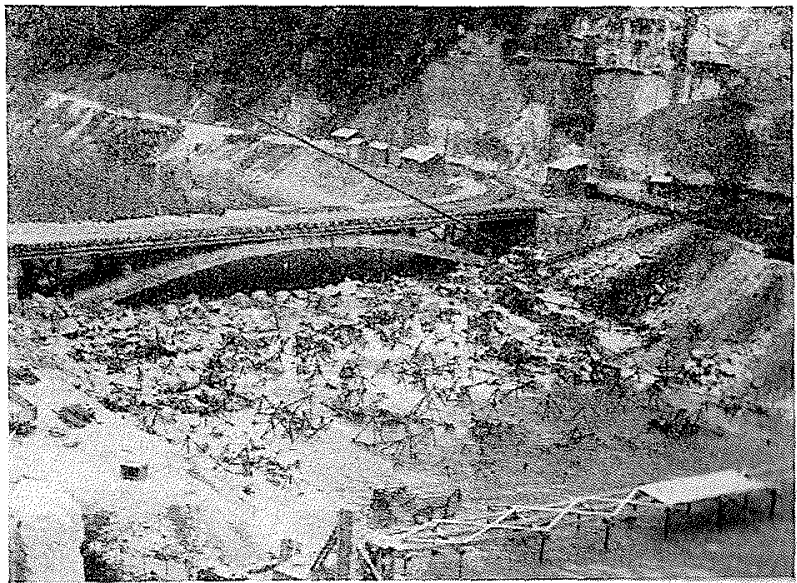

$17 b$

MASSI TERMIE, APRES OUVRRTURE DES DERIVATIONS

17 a) modelo

17 b) réalité 
grosseur intermédiaire entre ceux du lit et le tout-venant qui sera utilisé pour le barrage. Si ce dernier comprend une assez grande proportion de matériaux analogues en grosseur à ceux du lit, il ne sera pas nécessaire de donner à ce noyau de grandes dimensions.

Deuxième phase ou phase d'allonge. ment (fig. $15 \mathrm{~b}$, photo $12 \mathrm{~b}$ ). - On jette toujours, comme précédemment, le matériau tout-venant qui prend successivement la forme des trois premières configurations. On arrête cette opération lorsque le massif atteint sensiblement la longueur que doit avoir le massif final, dont le profil final doit être connu à l'avance, soit par calcul, soit après étude sur modèle. Au cours de cette opération, s'effectue le triage des matériaux déjà décrit précédemment. La pente de la zone à écoulement uniforme est faible. La zone de mise en vitesse se présente souvent avec un abaissement rapide de la ligne d'eau, suivi de quelques ondulations. Cette allure de la ligne d'eau est donnée par la différence de constitution de la surface de la zone de mise en vitesse. La crête est, en effet, formée par des gros blocs peu déviés lors de leur chute dans l'eau, tandis que l'on ne rencontre les éléments fins qu'un peu plus en aval. On conçoit alors que la granulométrie du mélange puisse conduire à une certaine disproportion entre l'élévation de la crête et la mise en vitesse nécessaire à l'entraînement des matériaux ve'rs l'aval. Ce phénomène n'a, en général, que très peu d'importance dans la conduite des opérations, car s'il est une cause d'amórce de brèches, ces dernières n'au'ront pas, en général, de graves conséquences avec ce mode de construction. Il donne à la surface de l'eau, un aspect ondulé caractéristique comme le montrent les diverses photos.

Troisième phase ou phase d'élévation (fig. $15 c$, photo $12 c$ ). - On utilise alors des engins spéciaux destinés à empêcher les gros blocs entraînés vers l'aval, de rouler sur le talus aval et ainsi de disparaître de la zone active du massif. Ces engins de formes les plus diverses peuvent être de deux types suivant qu'ils ofrrent aux gros bloes des appuis supplémentaires artificiels ou qu'ils produisent un ralentissement de la vitesse du courant permettant l'arrêt des gros blocs.

Pour simplifier l'exposé nous poursuivrons notre description en prenant comme exemple les ralentisseurs ou « retards » employés pour la coupure du Rhòne à Génissiat. Ces « retards» sont constitués par des cornières métalliques assemblées suivant les arêtes d'un tétraèdre (I).

(1) Cette techricule d'emploi des tétmèdres et d'tue facon plus querale, des retards, a fait l'oljet du brevet No 859.709 pris con

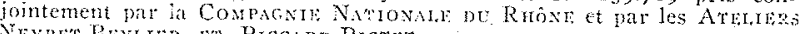
Neyret-Brylter fit Piccard-Brcters.
A un sommet de chaque tétraèdre est attaché un câble terminé lui-même à l'autre extrémité par une masse mélallique formant corps mort.

L'opération, qui consiste à faire élever le massif sans l'allonger, est alors conduite de la manière suivante : on dispose une première ligne de tétraèdres à l'extrémité aval de la zone à écoulement uniforme. Pour cela, on jette les corps morts sur le talus amont et ensuite les tétraèdres en aval de la crête du massif, les tétraèdres sont emportés par le courant jusqu'au bout du câble, dont les longueurs ont été préalablement réglées pour que les tétraèdres s'arrètent suivant la ligne voulue. Cette première ligne de tétraèdres étant mise en place, on jette des matériaux tout-venant. Sous l'effet du triage, les gros bloes arrivent à l'aval au droit des tétra. èdres, où ils s'arrêtent, car les tétraèdres ont été mis en nombre suffisant pour provoquer le ralentissement nécessaire du courrant. La zone des tétraèdres est alors extrêmement rugueuse, car à la rugosité du tétraèdre s'ajoute toute la rugosité des gros blocs; la pente d'équilibre que prend cette partie est donc très forte. Il en résulte le dépôt sur toul le massif en amont d'une certaine couche de matériaux. Cette cou. che étant formée, on procède à la mise en place d'une nouvelle ligne de tétraèdres en amont de la première et au lancement d'une nouvelle quantité de tout-venant. Comme précédemment, les gros éléments viennent former une pente raide au droit des tétraèdres, permettant le dépôt d'une nouvelle couche de matériaux sur le massif. On continue ainsi l'opération jusqu'à la dernière ligne de tétraèdres que l'on situera un peu en retrait de la ligne de lancement des blocs.

Le massif, pendant sa phase courante de construction comporte donc, en partant de l'aval :

$1^{\circ}$ un talus aval : celui qui a été obtenu au cours de la deuxième phase avec les matériaux tout-venant seuls.

$2^{\circ}$ une zone à écoulement uniforme a forte pente dont la surface très rugueuse est constituée par les tétraèdres et des gros blocs analogues à ceux qui, dans la deuxième phase, venaient former le talus aval.

$3^{\circ}$ une zone à écoulement unifome à faible pente constituée par les élénents fins et moyens du tout-venant triés par grosseur comme dans la deuxième phase.

4" une zone de mise en vitesse semblable à celle de la deuxième phase.

5 "un talis amont toujours formé par les plus gros blocs qui tombent en amont de la crête, cela si des dispositifs spéciaux n'ont pas été prévus pour remédiér à cet inconvénient.

On voit que la zone à forte pente est celle qui provoque l'élévation du massif. Cette élévation 
est obtenue grâce à la rugosité des gros éléments du tout-venant et à l'allongement vers l'amon de cette surface rugueuse, à forte pente. Le ròle de remplissage est, au conlraire, joué par les éléments moyens et fins qui forment la zone à faible pente.

L'art dans la conduite des opérations réside alors dans le dosage des lancements des tétraèdres et des matériaux. Chaque ligne de tétraedres doit, en effet, permettre une certaine élévation, c'est-à-dire le dépôt d'une certaine couche de matériaux dont il convient de connaître le volume.

Les différents profils que prend le massif peuvent ètre calculés, car les zones à forte ei faible pente du massif sont constituées par des. matériaux que l'on peut considérer comme triés.

Connaissant la granulométrie du tout-venant et d'après les volumes nécessaires au talus amont et aux deux zones à forte et faible pente, on peut connaître le diamètre moyen des matériaux constituant chaque zone et faire les calculs du profil en supposant que chaque zone est constituée uniquement par des matériaux ayant ces diamètres moyens. On peut avoir une plus grande précision en faisant plusieurs approximations successives.

Le nombre de "retards " à employer et la détermination de rugosité propre est plus complexe. On aura intérêt, en général, à utiliser un modèle réduit.

Remarques diverses sur cette technique - La forme prise par le massif est telle que les conséquences des amorces de brèches dans la crète sont bien moins grandes qu'avec la construction sans emploi de « retards ».

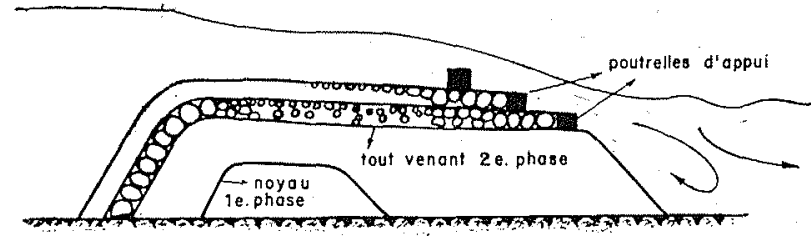

Fig. 16

L'ancrage des « retards 》 permet une meilleure résistance du massif lors des augmentations de débit ou des abaissements de niveau aval. En efret, une forte partie de l'efrort tangentiel supplémentaire exercé par le courant sur le massif est alors absorbée par ces ancrages, tandis que dans la construction ordinaire, les matériaux, à la limite de leur équilibre, ne peuvent supporter une augmentation d'effort tangenliel, ce qui amene les remaniements obligatoires du profil.

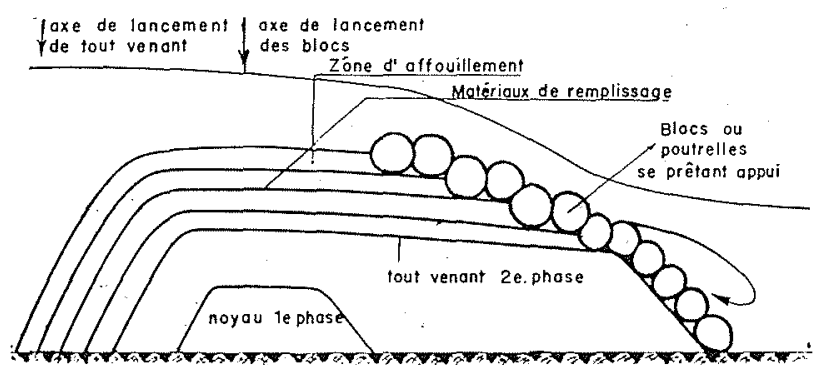

Fig. 17

- Dans la technique employée dans la coupure du Rhône, les câbles d'ancrage sont noyés dans la masse du massif peu après leur mise en place ; ils sont alors protégés contre le choc des pierres.

- Ponr diverses raisons, on aura intérêt à utiliser des câbles souples très résistants.

- Pour obtenir un meilleur rendement du tout-venant, on peut facilement imaginer un tremplin de lancement des blocs qui permette aux plus gros bloes de tomber plus en aval que les petites (grille inclinée formée de barreaux divergenls); on peut arriver ainsi à constituer le talus amont avec des éléments fins et à avoir la totalité des gros blocs dans la zone active. Un résultat sensiblement analogue sera obtenu avec deux lignes de lancement des matériaux.

- Divers engins autres que des tétraèdres peuvent être employés comme « retards », ils seront, en général, formés par un assemblage très ajou'ré en fers profilés du commerce ou en bois. Les modes de mise en place et d'ancrage peuvent être également très divers.

On peut aussi utiliser des engins qui agissent non par ralentissement des vitesses, mais par l'appui qu'ils offrent aux gros blocs. La fig. 16 montre un exemple de ce type d'engin; ce sont des poutres transversales saillantes.

- Le double effet de «ralentisseur et appui» peut également être recherché.

-- Un cas extrême est représenté par la fig. 17, la zone à forte pente est constituée uniquement par les engins eux-mêmes, qui dans le cas de la figure sont de gros blocs triés. Cette dernière construction est assez délicate, en raison des affouillements qui se produisent a la limite des deux zones; elle nécessite de plus une précision plus grande dans le dosage de lancement des deux catégories de matériaux.

\section{COUPURE DU RHONE A GENISSIAT}

Les travaux provisoires d'assèchement du lit du Rhône, pour la construction du barrage de Génissiat comprenaient, après le percement de deux galeries de dérivation souterraines, une dans 
chaque rive, la construction d'un massif de cotipure destiné à faire passer le fleuve par ces galeries. Ce massif serait construit en lancant des pierres dans le courant, à partir de l'un des deux ponts de service situés respectivement ì lemplacement des futurs batardeaux amont et aval.

La Compagnie Nationale du Rhône confia l'étude des problèmes hydrauliques relatifs a ces travaux au Laboratoire Dauphinois d'Hydraulique qui, avec l'aide de modèles réduits, entreprit d'abor une étude générale de la construction des massifs en pierres jetées dans le conrant, qui fut bientôt limitée au cadre fixé par les conditions particulières au chantier de Génissiat.

Ces conditions étaient essentiellement : 10 regime du Rhòne, la capacité d'évacuation des galeries, les possibilités d'extraction et de transport des matériaux, les dispositions des ponts, lespace possible pour lesmassifs, etc...

Le problème fut donc préalablement examiné sous ses aspects les plus divers et ce n'est que lorsque la date de mise en service des galerias fut prévue avec assez de précision, qu'un projet définitif de coupure fut mis au point.

Cette date fixait, en effet, une des données essentielles, à savoir la gamme des débits probables du Rhône durant la construction du massif, sans toutefois en préciser les variations.

Les grandes lignes du projet données par la fig. 18 étaient les suivantes :

Deux massifs de coupure seraient construits simultanément avant la mise en service des galeries el élevés à une cote telle que ces massif: émergent lorsqu'on ouvrirait une première galeWie, le clébii du Rhòne étant $300 \mathrm{~m} 3 / \mathrm{sec}$.

Le lancement des pierres s'effectuerait depuis la bordure aval de chacun des deux ponts.

Les matériaux utilisés seraient du tout-venani $(0-50 \mathrm{~kg})$ pour le noyau de la première phase et pour les autres phases des déblais de carlière $(0-300 \mathrm{~kg})$, avec emploi de tétraèdres pour raidir la pente et produire une élévation de niveau suirant la technique décrite précidem. ment. Les tétraèdres de 2.25 d'arètes seraient constitués par un assemblage de cornières $70 !$ $70 / 7$.

Les dimensions du massif aval, prévu par les études sur modèle réduit, étaient : Iongueur, 60 motres environ; largeur, 50 m.; hauteur, au-dessus du lit de la rivière au moment de l'ouverture de la première galerie, 9 mètres environ.

La construction du batardeau amont effectues avee les mêmes matériaux, mais sans tétraèdre, devait répondre à plusieurs buts :

-n uliliser l'énergie du courant d'eau poü la mise en place des matériaux du batardea amont : il suffirait, en effet, de basculer les matériaux dans le Rhòne depuis le pont amont.

- régulariser l'exploitation de la cariere, gagner du temps sur l'achèement des batardeaux, augmenter légèrement la dénivellation totale entre l'amont et l'aval.

L'époque de la construction faisait prevoir un débit du Rhòne pouvant varier de 140 à 800 $\mathrm{m} 3 / \mathrm{sec}$ avec un débit moyen probab!e de 300 $\mathrm{m} 3 / \mathrm{sec}$.

La photo n" 13 montre la disposition générale du chanlier. Vue de l'amont, on remarque : les entrées des dérivations, les deux ponts, la carrière su'r la rive droite, avec ses pelles de chargement; le train et sa voie a sens unique se bouclant par les rives et des deux ponts à parti? desquels les wagonnets étaient directement basculés dans la rivière.

Ce projet concu à partir des études préliminaires, avait été mis au point en détail ave? l'aide de deux modèles réduits; un modele en: canal vitré sur lequel fut éludié au $1 / 10^{\circ}$ la constiuction d'une tranche de chacun des massifs et un modèle d'ensemble des lieux à l'échelle $1 / 40$ (photo $n^{\prime \prime} 14$ ).

I.e mode operatoire complet fut détermine dans ses moindres détails : formation du noyau de protection du fonci, allongement du massió,

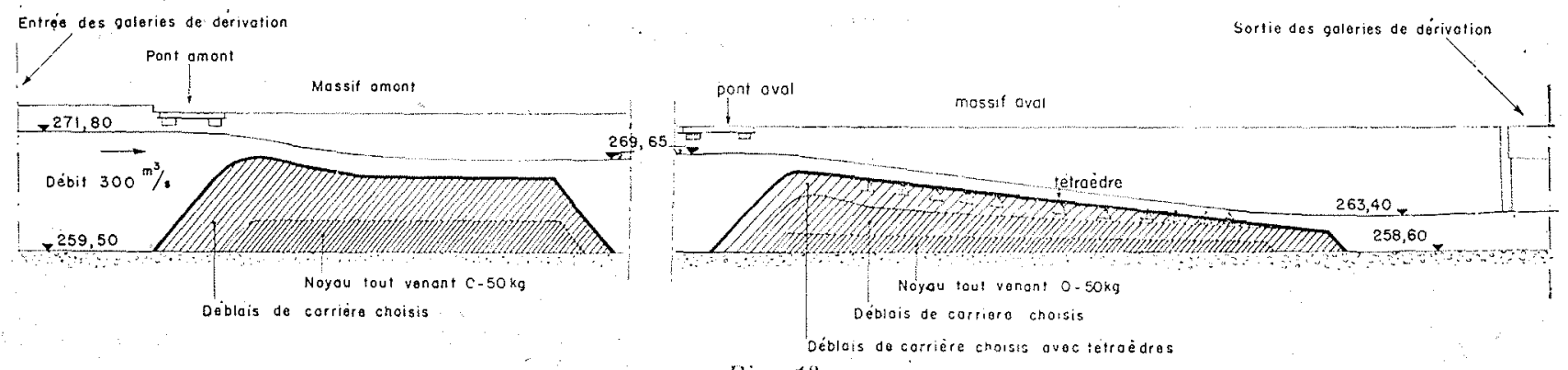

Fig. 18 
dosage des jets de matériaux el tétraèdres, protection des berges, effet des arches des ponts, formation des brèches, effet des fluctuations de débit, etc.

L'exécution réelle des massifs suivit presque exactement ces prévisions. Les photos de la page 43 montrent diverses phases de construction du massif aval sur le modèle et dans la nature.

Il y eut, au milieu de la phase d'élévation du massif, une crue de $600 \mathrm{~m} 3 / \mathrm{sec}$. Comme cela avait été indiqué très exactement par le modèle, la crête du massif s'est alors écrètée d'un mètre, sans que la partie aval subisse de remanie- ments : de mème des diminutions de débit ont produit, comme prévu, des allongements du massif à l'aval.

Finalement, la différence entre le volume des matériaux utilisés pour la coupure réelle et celui qu'avait donnó le modèle, s'est trouvée être inférieure à l'erreur de mesure de ces volumes.

Le nombre tolal des tétraèdres employés, 500 environ, pour le massif et la protection des berges a été également sensiblement le même qua celui prévu, si l'on y retranche ceux qui, perdus par rupture des căbles d'ancrage, ont dù être remplacés.

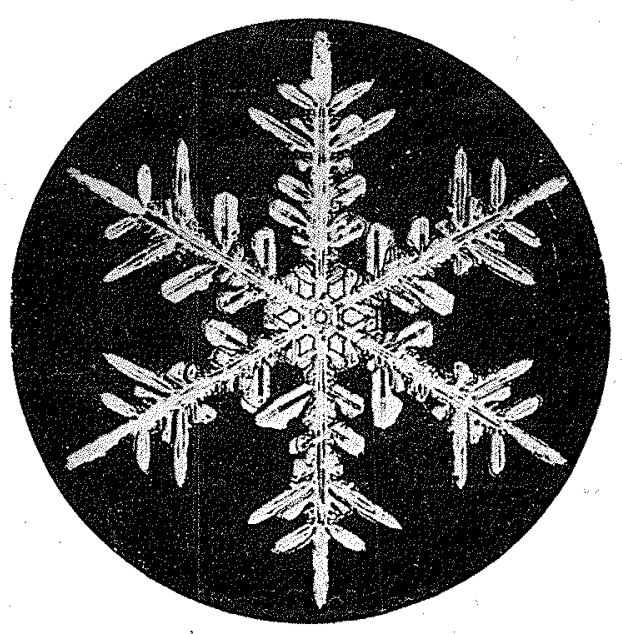

Au moment de motre sous presse, nous apprenons la mort de M. René Colomb, Directeur du Secteur d'Equipement de l'Electricité de France, Arve-Arc.

M. Coromb avait bien voulu aider La Houille. Blanche dans son démarrage, et le numéro de reprise de notre Revue présentait à ses lecteurs, un arlicle sur " La Reconstruction de la centrale de Bissorte ", auve personnelle de M. Colomb, qui avait tenu à préfacer lui-mème le travail de son collaborateur, M. L. Pousse, Directeur des travaux de reconstruction de la centrale.

Dans notre prochain numéro, nous relracerons la vie de celui qui fut et restera dans nos mémoires, un des plus grands réalisateurs de la technique hydraulique irangaise.

Que Mme R. Coloms veuille hien trouver, ici, lexpression de nos condoléances emues, et que les collaborateurs de son mari sachent combien nous participons au deuil qui les prive d'un tel chel. 